\section{LA-UR- $3-958$}

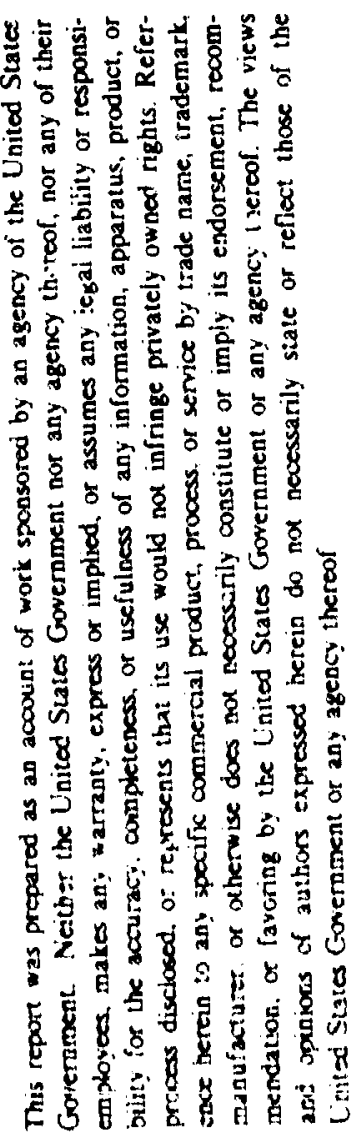

\section{Mrimis}

IEUTION OF IHIS OCCIIMEN IS IIMLIMITED

\section{Los Alamos}

NATIONAI IABORATORY

$$
\because C R F 9.544165-1
$$
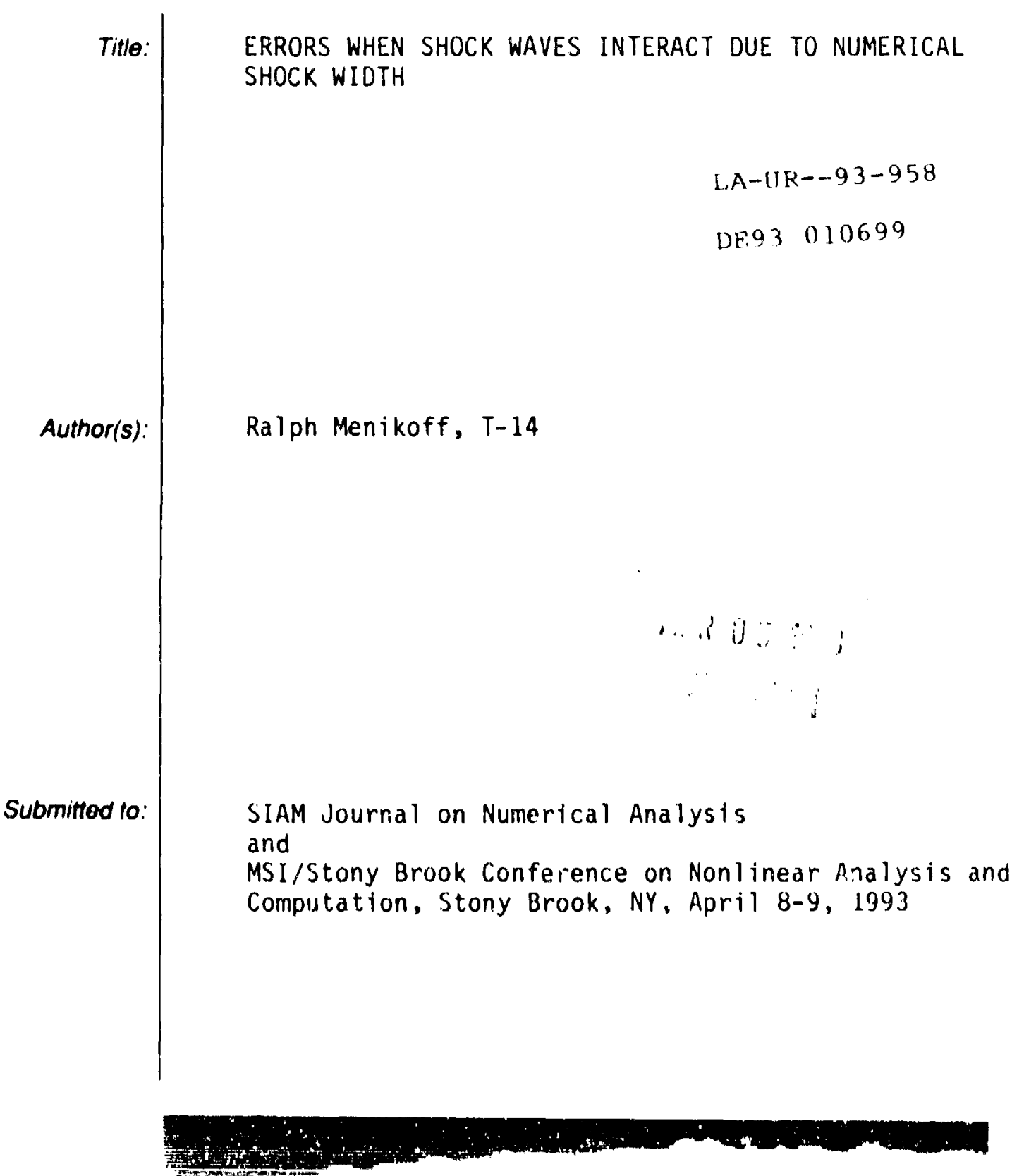

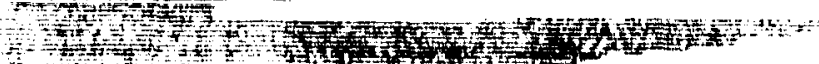

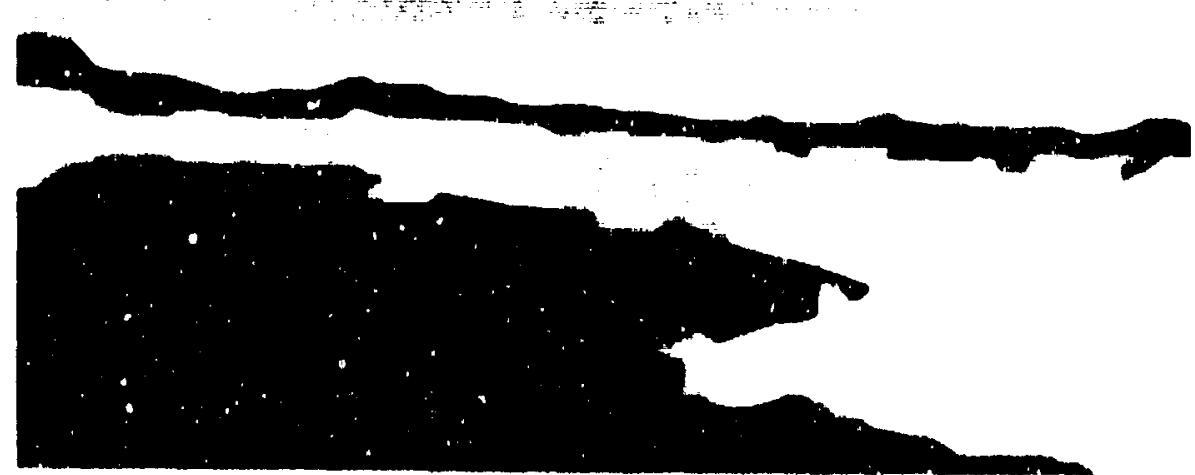




\title{
Errors when Shock Waves Interact due to Numerical Shock Width*
}

\author{
RALPH MENIKOFF \\ Theoretical Division \\ Los Alamos National Laboratory \\ Los Alamos, NM 87545 \\ (send correspondence to Ralph Menikoff: (505) 667-7761, FAX (505) 665-4055)
}

\begin{abstract}
A simple test problem proposed by Noh, a strung shock reflecting from a rigid wall, demonstrates a generic problem with numerical shock capturing algorithms at boundaries that Noh called "excess wall heaing." We show that the same type of numerical error occurs in general when shock waves interact. The underlying cause is the non-uniform convergence to the hyperbolic solution of the inviscid linit of the solution to t'.te PDEs with viscosity. The error can be understood from an analysis of the usymptotic solution. For a propagating shock, there is a difference in the total energy of the parabolic wave relative to the hyperbolic shock. Moreover, the relative energy depends on the strength is the shock. The" error when shock waves interact is due to the difference in the relutive energies betwern the incoming and outgoing shock waves. It is nnalogous to a phase shift in a scoutteriug mntrix. A conservative differencing scheme correctly describes the Hugoniot jump conditions for a stendy propagnting shock. Therefore, the error from the asymptoticy orcurs in the transicnt when the waves internct. The entropy error that ocrurs in the interartion region remains locenlized but does not dissipnte. A senling arkument shows that as the viscosity

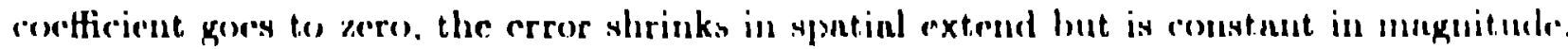
Noh's problem of the reflection of a shock from a rigial wall is cepuivalent to the symuretrie

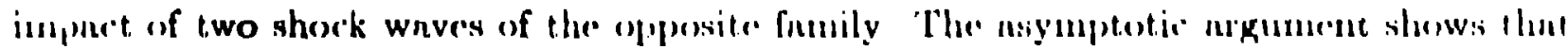

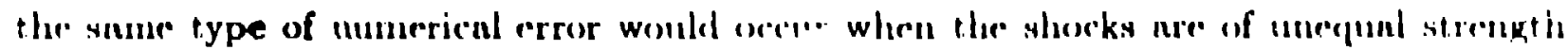
Thus, Noh's problest is indientive of $1 \mathrm{mum}$ and error thut arours when shoseks intermet due to the mumericnl shorek width.
\end{abstract}

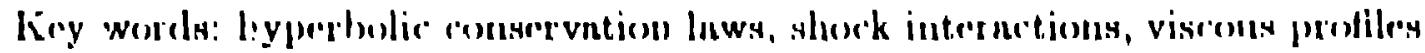

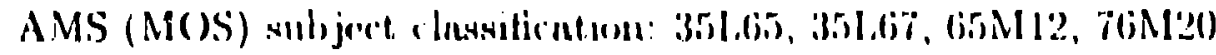

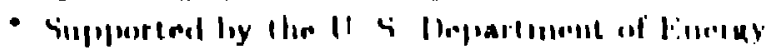




\section{Introduction}

The equations for ideal fluid tlow form a hyperbolic system of conservation laws

$$
\partial_{\mathrm{t}}\left(\begin{array}{c}
\rho \\
\rho u \\
\rho\left(\frac{1}{2} u^{2}+E\right)
\end{array}\right)+\partial_{x}\left(\begin{array}{c}
\rho u \\
\rho u^{2}+P \\
\rho\left(\frac{1}{2} u^{2}+E\right) u+P u
\end{array}\right)=\overrightarrow{0}
$$

where $\rho$ is the density, $u$ is the particle velocity, $E$ is the specific energy, $P(V, E)$ is the pressure and $V=1 / p$ is the specific volume. Dissipation only occurs across a shock wave and physically is accounted for by imposing the Rankine-Hugoniot jump relations across the shock discontinuity. Finite difference shock capturing algorithms are frequently used to obtain a numerical solution to the fluid flow equations. These schemes have a numerical dissipation that gives a shock wave a small width measured in grid cells, but in artificially large spatial width conpared to the typical shock width that physically occurs. The effect of the artificial shock width is largest when shock waves interact. To determine: the effect of the numerical shock width, we analyze the asymptotic solution for a simple: shock interaction when a viscous dissipative term is added to the ideal fluid equations.

The problem we rousicler in derail is a strong shock in in ideal gas retlecting from $n$ rigid wall. This is equivalent to the interaction between equal strength shosks of the opposite fnmily. It is similar to a test p:oblem Noh [3] introduced that exemplify errors in

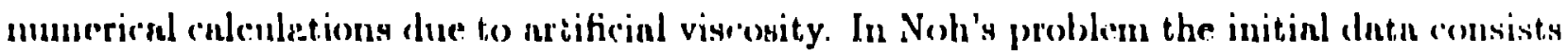
of a uniform stnte of cold gas with a constant velocity dirceted cowards n rigid wall. Its solution han a strong outgoing shock. Brenuse of the zoro initial sonnd spered, an analytir

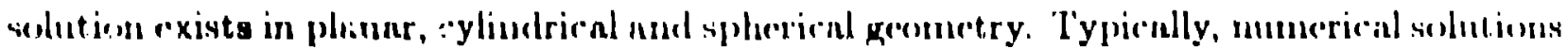

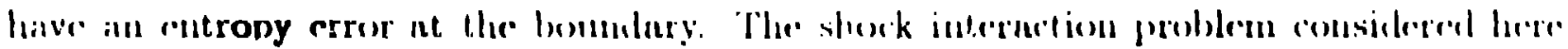

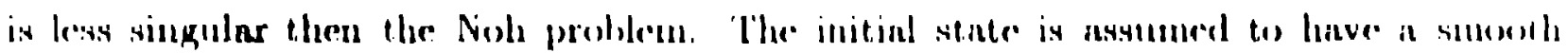

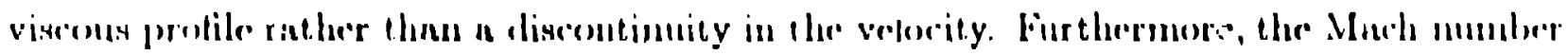

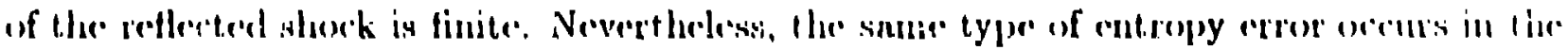

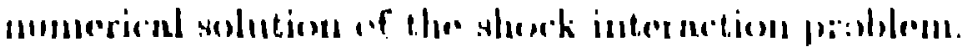

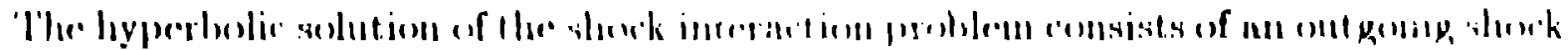

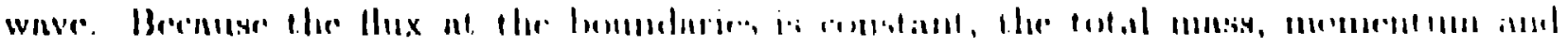


energy in the viscous solution have the same value as those in the hyperbolic solution. Wr. define the shock position of the viscous wave to have the same total mass and momentum as the hyperbolic shock wave. An important quantity in the asymptotic analysis is the energy of the viscous shock relative to the energy of hyperbolic shock.

We show that there is a shift in the relative energy between the incoming and outgoing waves. This implies that an entropy error must occur during the transient shock interaction. For a shock reffection, the transient take" place when the shock profile overlips with the boundary. After the transient, the entropy is frozen in place, i.e., convects along particle trajectories, and the error does not dissipate.

A scaling argument due to Noh shows that as the viscous coefficient goes to zero the entropy error decreases in spatial extent but not in magnitude. It implies that the convergence of the inviscid limit to the hyperbolic solution is non-uniform in regions where shocks have interacted.

\section{Asymptotics}

Let us consider a stendy state viscous shork wave. Suppose i! we wave is rigint facing nud propagnting with velocity o. Furthermore, let the reference points $s_{a}$ atud $r_{b}$ be in

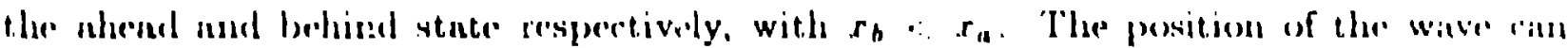

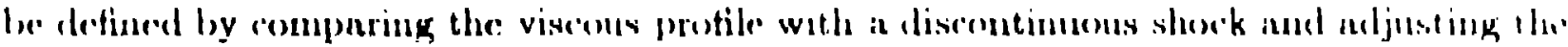
discontinuity such that the two wheres have tive sane total mass.

'The condition that the wavers have the same mass is given by

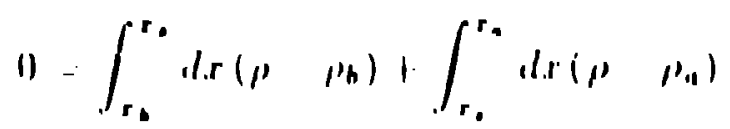

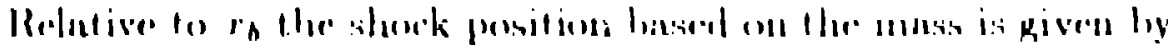

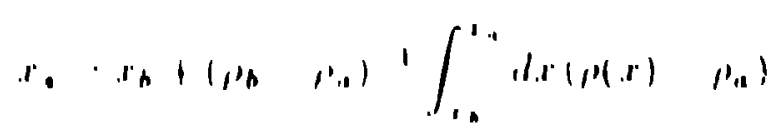


Similarly, the position of the wave could be defined by matrhing the total momuntum. T'1e shock position based on moinentum is obtained from Eq. (2.2) by replacing the mass density $\rho$ with the momentum density $\rho u$.

In steady state the mass Hux is everywhere constant

$$
\rho(u-\sigma)=m
$$

Hence, there is a linear relation between mass density and momentum density $\rho u=\rho \sigma+m$. Consequently the shock positions, based on either the 1.1ass or momentum of the waves, are the same.

One could also base the shock position on the total energy. However the energy density $E=\left(\frac{1}{2} u^{2}+E\right) \rho$ is not Galilean invariant. This would lead to a non-muigurness in the shork position. Instead, we detine the relative energy between the viscous protile and the discontinuons shock with the shock position based on mass

$$
\begin{aligned}
& \partial E^{r}=\int_{r_{0}}^{x_{1}} d \cdot c\left(\varepsilon-\varepsilon_{h}\right)+\int_{r_{0}}^{x_{0}} d x\left(E-\ldots \varepsilon_{10}\right) \\
& \because \int_{r_{b}}^{r_{a}} \operatorname{dlr}\left(\varepsilon-\varepsilon_{1 a}\right)-\left(x_{0}-x_{b}\right)\left(\varepsilon_{b}-\varepsilon_{a}\right)
\end{aligned}
$$

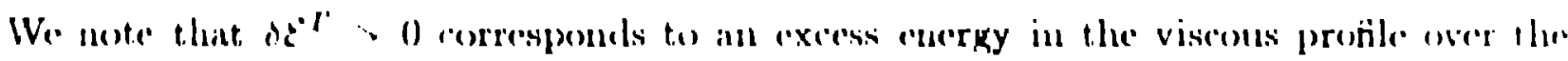
Iliscontimuouss shorek.

We next show that the relative energy is Cialilean inveriant nud hence well defined.

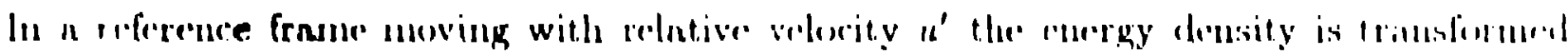

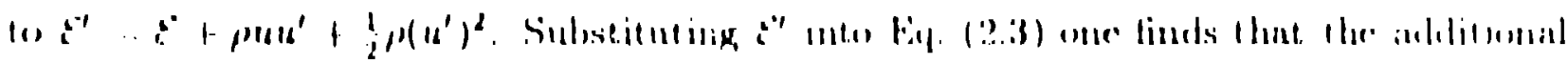

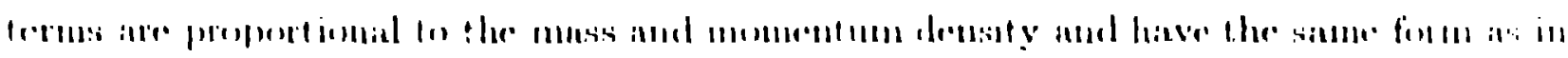

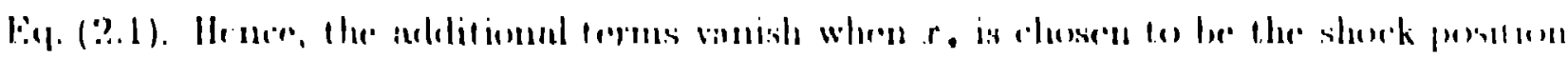

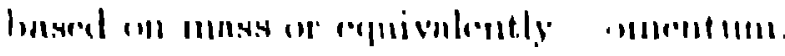




\section{Von Neumann.Richtmyer Viscosity}

Viscosity can be incorporated into the fluid equations by adding a viscous pressure onto the fluid pressure, $P \rightarrow P+Q$ in Eq. (1.1). We analyze the viscous fluid equations using a von Jeumann-Richtinyer viscosity [2] and an ideal gas equation sf state. The von Neumann-Richtmyer viscosity is defined by the viscous pressure

$$
Q= \begin{cases}C_{\nu} \rho \ell^{2}\left(\partial_{x} u\right)^{2}, & \text { if } \partial_{x} u<0 \\ 0, & \text { otherwise }\end{cases}
$$

where $C_{\nu}$ is a dimensionless viscosity and $\ell$ is a length scale proportional to the shock width. Without loss of generality we can set $C_{b^{\prime}}=1$. For an ideal gas

$$
P V=(\gamma-1) E
$$

with $\gamma>1$.

In this case, there is an exact analytic formula for the viscous profile of a shock wave $[4]$. Let $\sigma$ be the shock velocity and the variable

$$
w=\left(\frac{\gamma+1}{2}\right)^{\frac{1}{2}} \cdot\left(\frac{x-\sigma t}{\ell}\right)
$$

be a sealed length relative to the shork front. Then the viscous pretile is given by

$$
\begin{aligned}
& I^{\prime}(u)=\frac{1}{2}\left(V_{a}+V_{b}\right)+\frac{1}{2}\left(V_{a} \cdot V_{b}\right) \sin (w)
\end{aligned}
$$

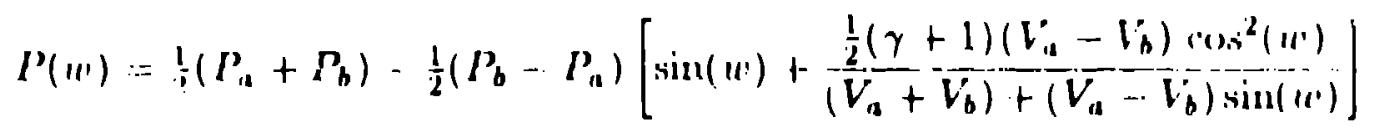

$$
\begin{aligned}
& Q(u)=1 / 4(\gamma+1)\left(P_{b}-P_{a}\right)\left[\frac{\left(V_{a}-V_{b}\right) \cos ^{2}(u)}{\left(V_{a}+V_{b}\right)+\left(V_{b}-V_{b}\right) ! \operatorname{in}(u)}\right] \\
& u(w)=\sigma-m l(w)
\end{aligned}
$$

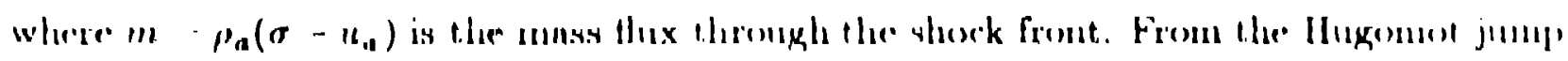

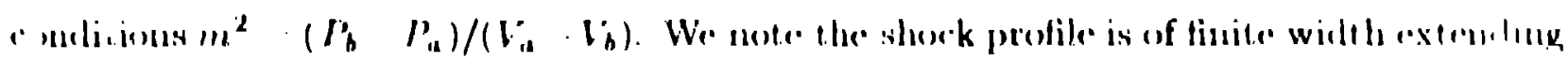

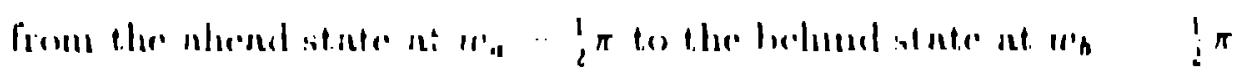

The shorek position hower! on must is yiven hy

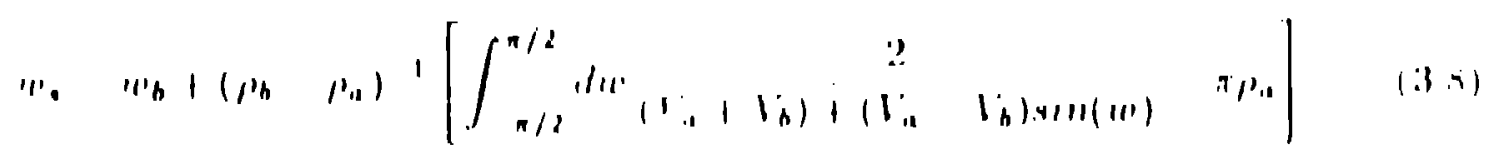


The integral is of the form evaluated in the appendix. It can be simplified to give

$$
w_{0}=w_{b}+\pi\left(\frac{\eta \frac{1}{\frac{1}{2}}-1}{\eta(\eta-1)}\right)
$$

where $\eta=V_{u} / V_{b}$ is the compression ratio of the shock. We note the limiting cases: for a weak shock $\eta \rightarrow 1$ and $w_{s} \rightarrow 0$ while for a strong shock $\eta \rightarrow(\gamma+1) /(\gamma-1)$ and $-\frac{1}{2} \pi<w_{s}<0$

It is convenient to calculate the relative energy of the shock profile in the rest frame of the shock front, i.e., $\sigma=0$. In this case the kinetic energy is $\frac{1}{2} \rho u^{2}=\frac{1}{2} m^{2} V$ and the energy density can be expressed as

$$
\mathcal{E}=\frac{1}{2} m^{2} V+(\gamma-1)^{-1} P
$$

Substituting this expression into Eq. (2.3) for the relative energy we cobtain

$$
\begin{aligned}
& \partial \mathcal{E}^{T}=\left(\frac{2}{\gamma+1}\right)^{1} \ell\left[\frac{1}{2} m^{2} \int_{-\pi / 2}^{\pi / 2} d w V(u)+\frac{1}{\gamma-1} \int_{-\pi / 2}^{\pi / 2} \text { iw } P(u)\right) \\
& \left.-\pi \varepsilon_{a}-\left(u^{\prime}-u_{b}\right)\left(\varepsilon_{b}-\varepsilon_{a}\right)\right]
\end{aligned}
$$

'The integrals can be evaluated with the formulare in the nppendix.

$$
\begin{aligned}
& \int_{-\pi / 2}^{\pi / 2} d w l(w)=\frac{1}{2} \pi\left(V_{a}+V_{b}\right) \\
& \int_{-\pi / 2}^{\pi / 2} d w P(w)-\frac{1}{2} \pi\left[\left(P_{a}+P_{b}\right)-\frac{1}{2}(\gamma+1)(\eta t+-1)^{2}(\eta-1)^{-1}\left(P_{b} \ldots P_{a}\right)\right)
\end{aligned}
$$

After steraghtforwnel algebraic unuipulntion, we obluin for the reiative cuergy

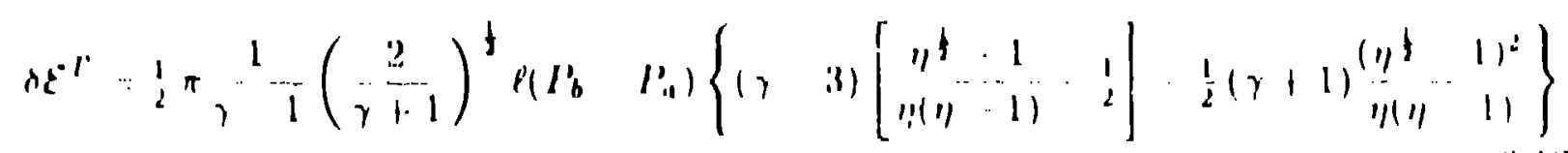

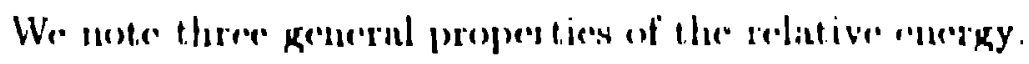

(1) $i \varepsilon^{-l}$ in a fimetion of the shosek width.

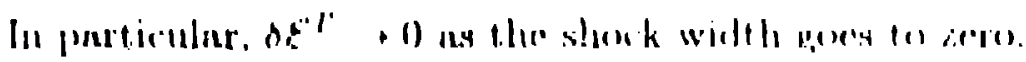


(2) $\delta \mathcal{E}^{T}$ is a function of the shock strength.

For weak shocks $\delta \mathcal{E}^{T} / \ell \sim\left(P_{b}-P_{a}\right)^{2}$ and for strong shocks $\partial \mathcal{E}^{T} / \ell \sim\left(P_{b}-P_{a}\right)$.

(3) $\delta \varepsilon^{T}$ varies with $\gamma$, and hence the equation of state.

This is a consequence of the fact that the viscous pressure depends only on the density and velocity, hence the shock profile depends on the equation of state.

These important properties are expected to be true for any reasonable viscosity and equattion of state.

\section{Example of Reflected Strong Shock}

The effect of the shock width on a shock interaction can be seen in the simple case of a strong shock retlecting from a rigid wall. To compare the viscous solution with the hyperbolic solution, we compute the difference in the relative energy between the incoming shock and the outgoing shock, $\Delta \mathcal{E}^{T}=\delta \mathcal{E}_{r}^{T}-\delta \mathcal{E}^{T}$. We note that $\Delta \mathcal{E}^{T}>0$ corresponds to a net excess energy in the viscous shock profiles compared to the hyperbolic shocks.

Let the pressure behind the incoming shock be $P_{\mathrm{s}}$. The compression ratio of at sirong shock is $\eta_{0}=(\gamma+1) /(\gamma-1)$. Tla reflected shork is characterized by its pressure ratio,

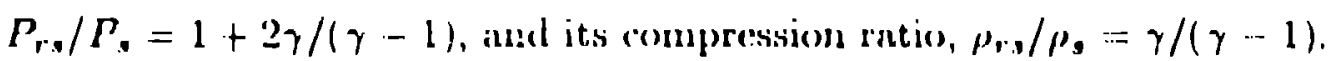

The :scale for the relative conergies is e $=\frac{1}{2} \pi \frac{1}{\gamma-1}\left(\frac{2}{\gamma+1}\right)^{t} P P_{9}$. Substituting the values for the pressure and compression atio into E. E. (3.11) we obtaia for the relative energies

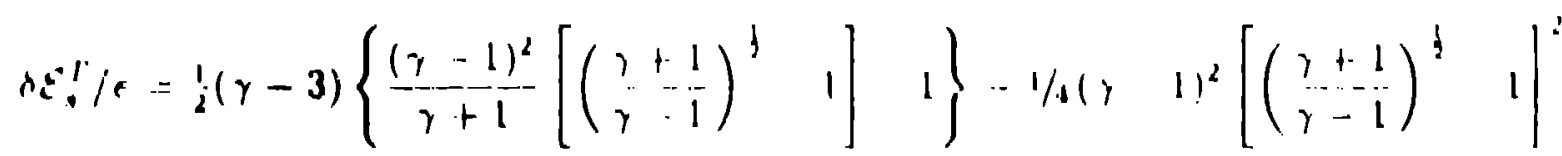

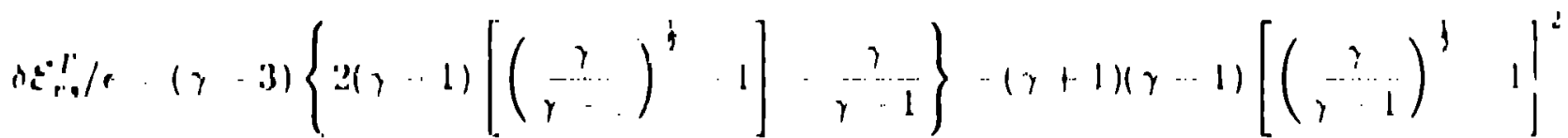

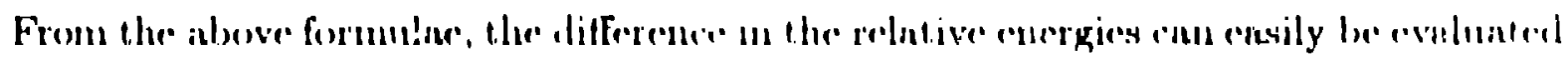

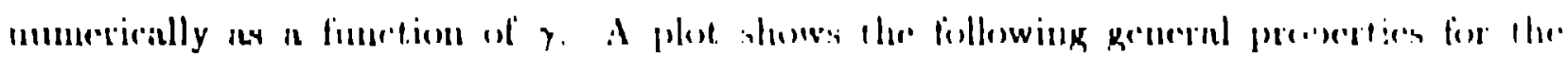

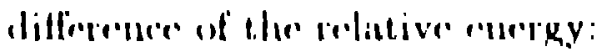


(1) $\Delta \mathcal{E}^{T} \rightarrow \infty$ as $\gamma \rightarrow 1$

This singularity is due to the singularity in the compression ratio at $\gamma=1$.

(2) $\Delta \mathcal{E}^{T}=0$ at $\gamma \approx 2.4$

(3) The ininimum value of $\Delta \varepsilon^{T} \approx-0.34$ occurs at $\gamma \approx 4.65$

(4) $\Delta \mathcal{E}^{T} \rightarrow 0$ as $\gamma \rightarrow \infty$.

We note that in general $\Delta \mathcal{E}^{T}$ is not zero.

The constant flux ahead of the outgoing wave can be accounted for by comparing the position of the shuck in the viscous solution to that of the hyperbolic solution. The shift in the energy of the viscous shock profiles implies that a steady state outgoing wave can not simultaneously satisfy the flux relations for mass, momentum and energy. Instead, the shock interaction must result in a transient. The transient occurs on both a fast and slow time scale and results in an entropy error when comparing the viscous solution to the hyperbolic solution.

Over the fast time scale, (shock width)/(shock velocity), the viscous pressure smoothes out any discontinuity in the non-degenerate or acoustic modes. This is important when the positions of the incoming and outgoing shock waves are within a few shock widitus of the wall. The pressure and particle velority rapidly equilibrate towards the values of the hyperbolic solution as the incoming shock profile changes to the outgoing profile. On the slow time scrale, the viscous solution is cluse to the solution to the Riemann problem and the outgoing shork protile approaches its steady state solution.

On the slow time scale, the shift in rnergy is small compared to the total merng behind the shock. The energy mismatch in the shock profiles an be distributed over the region between the wall and the shork frome by acoustic waves. 'The entropy cror at the whork front is small and further decrense as $1 / t$ for large $t$. This is $n$ consecpuence of the

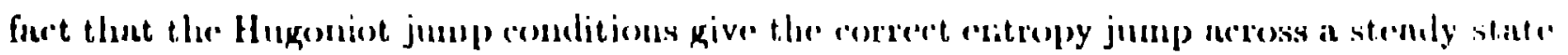
shock profile indeperaclent of the form of dissipntion.

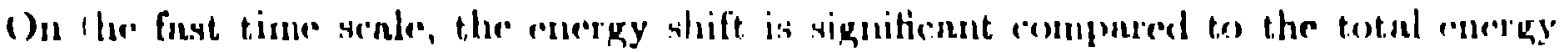
in the shorek profile. This results in a signitione entropy error in the internetion rexion 
during the transient in which the shock profiles change. After the pressure and particle velocity have equilibrated, the viscous pressure in the interaction region approaches zero and the subsequent chang $\mathrm{g}$ in entropy is negligible. Without heat conduction which would give rise to diffusion of entropy, the entropy error is frozen into the particle trajectories. Thus, the bulk of the entropy error from the interaction is confined to within a few shock widths of the wall.

Let us consider in more detail the interaction region for the case when $\Delta \mathcal{E}^{T}>0$. Vear the wail the outgoing viscous wave must have a deficit in energy equal to $\Delta \mathcal{E}^{T}$ in o:-der to compensate for the energy difference in the shock proxies. Because the wall causes the particle velocity to go to zero, the energy density reduces to $\mathcal{E}=\rho E=P /(\gamma-1)$ and is proportional to $P$. When the reflected wave has propagated a couple of shock widths, the pressure has approximately equilibrated to the value behind the outgoing hyperbolic shock. In order to conserve total energy, the viscous shock front must be slightly behind the hyperbolic shock front. Then to conserve mass, on average $\rho$ must be above the value for the hyperbolic shock. Since $P$ is approximately constant, a high value for $\rho$ implies on average the entropy $S \propto \log \left(P / \rho^{\gamma}\right)$ is low.

At the wall, the pressure rise is more characteristic of a single strong shock then a double shock. Since the entropy is greater for a single strong shock then for two sequential shocks to the same final pressure, right at the wall we expect the entropy to be high and the density to be low. This implies there is an oscillation in the density and entropy in the vicinity of the wall. The pressure and density determine the specific energy through the equation of state. At the wall, a low value of $\rho$ results in a high value of $E$. This agrees with the results of numerical calculations and is what Noh |3| called excessive wall heating, even though there is a clamped oscrillation in the energy about the value behind the hyperbolic shock.

Finally, to conserve total momentum the velocity profile overshoots and beiones slightly uegative immediately behind the viscous shock front. As the whve moves further uwny from the wall, the viscous profile: nore closely nppronches that of a stendy stiate

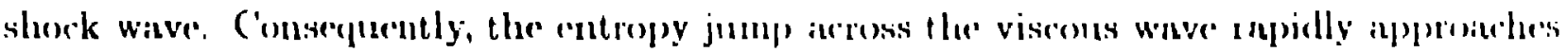


the value for the hyperbolic shock. As time progresses, further errors in entropy outside the interaction region are negligible.

We note that the initial data for . Noh's test prublem corresponds in effect to taking the relative energy of the incoming wave to be zerc. In this case, the energy difference for the interaction is $\Delta \mathcal{E}^{T}=\delta \mathcal{E}_{s}^{T}$. Again, in general $\Delta \mathcal{E}^{T}$ is not zero and an entropy error occurs from the transient interaction that forms the outgoing shock.

Finally, to understand the small distance it take for the shock to form and the pressure and velocity to equilibrate we estimate the magnitude of $\delta \mathcal{E}_{\mathrm{s}}^{T}$ relative to the energy in the shock profile. For illustrative purposes we assume $\gamma=5 / 3$. From Eq. (3.3) the shock width is $\Delta x=2.72 \ell$. The compression ratio of a strong shock is $\eta=(\gamma+1) /(\gamma-1)=4$. From Eq. (3.12), the energy ratio is $\delta \mathcal{E}_{\mathrm{g}}^{T} / \Delta x \mathcal{E}_{\mathrm{g}}=1 / 9$. Thus the energy in the shock profile will have a small effect on the shock interaction after the outgoing shock has propagated a couple of shock widths.

\section{Non-uniform convergence of Inviscid Limit}

One important consequence of shock interactions is that the convergence of the inviscid limit to the hyperbolic solution is non-uniform. This may be deduced through a scaling argument introduced by Noh [3].

The inviscid fluid equations are scale invariant. Scaling space and time amounts to it choice of units. Viscosity iatroduces a length scale which breaks the invarian:e. However. uncler scaling, the viscous pressure is multiplied by th constant. Therefore, by scaling the coefficient of viscosity along with the length and time scales, the equations are again invariant A solution to the fluid equntions with the von Neumann-Richtmyer viscosity is invariant under the transformation $x^{\prime}=\alpha x, t^{\prime}=\alpha x$ and $C_{\nu}^{\prime}=\alpha^{2} C_{\nu}$. Furthermore. this transformation preserves velocity and hence the initial value clata. As $\alpha \rightarrow 0$, the entropy caror at the wall is constant in magnitude but decreases in spatial extent. Hence the inviscid limit for this case converges in $L^{1}$ or $L^{2}$ hut not in $L^{\infty}$. 
A shock reflecting from a rigid wall is equivalent to the symmetric collision of two shocks, i.e., equal strength shocks of the opposite family. The argument that the cause of the error is due to the asymptotic shift in the relative energy between the incoming waves and the outgoing waves implies $t$. at. the fact that the incoming waves are of eyual strength is not important. Hence, shock interactions in general will result in non-uniform convergence of the inviscid limit.

\section{Effect of Source terms}

Noh also has a version oi the shock reflection test problem in cylundrical and spherical geometry. This introduces an additional effect on shock propagation due to geometrical source terms.

The geometrical source terms are singular at the origin. Consequently, as the shock approaches the origin the source terms become comparable in magnitude to the viscous dissipation within the shock profile. When this occurs, the conservation form of the equations no longer implies the Hugoniot jump condition across a shock.

A real effect in which the Hugoniot jump conditions are modified occurs for detonation wayes [1]. In this case the competition between chemical reactions and geometrical source terms gives rise to the curvature effect in which the detonation velocity depends on the curvature of the shock front. An artificiai!y large numerical shock width and geometric source terms can have a similar effect near the origin.

An idea converging shock, from the Gurdeley similarity solution, is singular at the origin. The shock width provides a length scale which regularizes the singularity when the shock reflects from the origin. After reflection there are large gradients behind the shock front. The shuck has to propagate a sutticient alistance from the origin in order for the gradients behind the shock to be small compared to those in the shock profile. This is a aecessury condition for the Hugoniot jump conditions to apply arows the niurk independent of the form of dissipation. 
Thus, when source terms or gradients behind the shock front are large compared to the dissipation within the shock profile, the viscous solution can differ signiticantly: from the hyperbolic solution. Again the error is in the entropy and is expected to be localized. Finite difference shock capturing algorithms have an artificially large shock width. Numerical solutions with schemes that have the smallest shock width will minimize errors of this type and be closest to the hyperbolic solution

\section{Conclusion}

We have analyzed the problem of a strong viscous shock reflecting from a rigid wall. For the von Neumann-Richtmyer viscosity, we have shown that the same type of entropy error occurs as in Noh's test problem. The error is due to the difference in energy relative to the hyperbolic solution of the viscous profiles for the incoming and outgoing shock waves. A scaling arguments shows that as the viscous coefficient goes to zero the entropy error decreases in spatial extent but not in magnitude. Furthermore, the entropy error is convected with the fluid and does not dissipate.

From the asymptotic energy argument, we expect the same behavior to cocur for an arbitrary shock interaction with any dissipative mechanism that results in a non-zero shock width, provided there is no heat conduction to diffuse entropy. The dissipation may correspond to a term added to the hyperbolic PDEs, e.g., an artificial viscosity, or "an be: numerical in nature, e.g., resulting from truncation errocs in the differencing schome ar a Riemann solver used in the Godunov method. The fact that hyperbolic finite differenc: schemes deliberately underresolve the shork profile is not critical. The iruncation reror:s merely introduce an oscillation in the shock profile as the position of the shork front propagates between krid points.

The entropy error when viscous shork protiles infernet implies a non uniform comber gence of the inviscid limit to the hyperbolic solution. Non maform convergence an be expected at the shock front. An adelitional uon - uniformity cnu orour in a region in which 
the solution is smonth resulting from a shock interaction that occurred in the region's past history.

A more severe form of this entropy error occurs when a shock wave is incident on a material interface or contact. For materials with different equations of state or when the contact is a discontinuous change in zoning, there can be a large transient resultin; from the change in profiles for the incident, transmitted and reflected shock waves. In Lagrangian algorithins the effect is partially ameliorated by choosing the grid such that the wave speed in units of zones per time step is the same for the outgoing shocks on each side of the interface. However, the minimal error is similar to that which occurs for the shock intcraction discussed here.

In more complicated fluid flows, additional errors can result from the inhomogeneities caused by the entropy erzor from shock interactions. For example, subsequent shock waves will scatter off the inhomogeneities and spread the spatial extent of the error. This effect is partially ameliorated by the fact tiat shock heating raises the sound speed. Hence subsequent reflected shocks have a lower Mach number and the additional entropy errors they cause decrease as the shocks weaken. Another example occurs in an unstable two dimensional flow. The inhomogeneities from shock interactions can be the seed for a perturhation which leads to instability growth.

For some applications, the non-uniform convergence is important. One example is when comparing the calculated temperature at a wall to experimental data. The numerical entropy error from a reflected shock results in a high wall temperature which does not iissipate in time. Moreover, the calculated wall temperature does not improve under mresh refinement. Having understixud the cause, one can compenante for this error, (P., w, with sufficient resolution by nueraging over a small region in the vicinity of the wall. Anothur exumple is when the material is chemically reactive. in particular, for nu explosive " numerical hot spot caused by a shork interaction can initiaten detonation and grently offect the Aluid fow.

The spentind extend of the entropy error whers slocks interact is piriportionul to the shock width. 'Thus, this error is sinallent for those nurnorical acherne that minimize thr' artificin shock wirlth. In particular, this type of orror can be eliminated by usung " front. irncking ngorithn. 
Errors when Shock Waves Interact

March 4, 1993

Acknowledgment

I would like to thank my colleague Dr. Klaus Lackner for many enlighter.ing discussions on this subject. 


\section{Appendix: Evaluation of Integral}

The needed integrals can be evaluated by contour integration as follows. Suppose $a>b>0$ and $n$ is a non-negative integer. Let $z=e^{i x}$. Then the basic integral of interest can be expressed as

$$
\begin{aligned}
\int_{0}^{\pi} d x \frac{\cos (n x)}{a+b \cos (x)} & =\operatorname{Re} \int_{C_{0}} \frac{-i d z}{z} \cdot \frac{z^{n}}{a+\frac{1}{2} b(z+1 / z)} \\
& =\operatorname{In} \int_{C_{0}} d z \frac{z^{n}}{\frac{1}{2} b z^{2}+a z+\frac{1}{2} b}
\end{aligned}
$$

where $C_{0}$ is the arc of a unit circle in the upper half of the complex plane.

The denominator of the integrand on the RHS has two zeros located at

$$
z_{ \pm}=\left[-a \pm\left(a^{2}-b^{2}\right)^{\frac{1}{2}}\right] / b \text {. }
$$

These lie along the real axis with $z_{-}<-1$ and $-1<z_{+}<0$. Let $C$ be the path formed by closing the path $C_{0}$ along the $x$ axis but going around the pole at $z_{+}$in the upper half plane. By applying Cauchy's residue formulae we obtain

$$
\begin{aligned}
\int_{0}^{\pi} d x \frac{\cos (n x)}{a+b \cos (x)} & =\operatorname{Im}\left(\int_{C} d z \frac{z^{n}}{\frac{1}{2} b \bar{z}^{2}+a z+\frac{1}{2} b}-P V \int_{-1}^{1} d x \frac{x^{n}}{\frac{1}{2} b x^{2}+a x+\frac{1}{2} b}\right. \\
& =\pi \operatorname{llesidue}\left(z_{+}\right) \\
& =\frac{\pi}{\left(a^{2}-b^{2}\right)^{\frac{1}{3}}}\left(\frac{\left(a^{2}-b^{2}\right)^{t}-a}{b}\right)^{n}
\end{aligned}
$$

Using the symmetry of the sin and cos functions over a half cycle we note two spercial (atsey of the above formula

$$
\begin{aligned}
& \int_{-b \pi}^{1 \pi} d x+\frac{1}{a+b \sin (x)}=\int_{0}^{\pi} d x+a+\frac{1}{a+b \cos (x)} \\
& =\frac{\pi}{\left(a^{2}-b^{2}\right)^{1}} \\
& \int_{1 \pi}^{h \pi} d x \frac{\cos ^{2}(x)}{14+b \sin (x)} \quad \int_{10}^{\pi} d x \frac{\sin ^{x}(x)}{a+b \cos (x)} \\
& 1 \int_{11}^{\pi} \frac{1}{11}+\cos (2 x) \\
& b^{2}\left|a \quad\left(a^{2}-b^{2}\right)^{b}\right|
\end{aligned}
$$




\section{References}

[1] B. Bukiet and R. Menikoff, Detonation waves and the Front Tracking Method, Phys. Fluids A, 4 (1992), pp. 2070-2081.

[2] J. von Neumann and R. D. Richtmyer, A Method for the Numerical Calculation of Hydrodynamic Shocks, J. Appl. Phys., 21 (1950), pp. 232-237.

[3] W. F. NoH, Errors for Calculations of Strong Shocks Usirg an Artificial Viscosity and an Artificial Heat Flux, J. Comp. Phys., 72 (1987), pp. 78-120.

[4] R. D. Richtmyer and K. W. Morton, Difference Methods for Initial-Value Problems, Interscience, New York, 1967. 